\title{
TAUBERIAN THEOREMS AND STABILITY OF ONE-PARAMETER SEMIGROUPS
}

\author{
W. ARENDT AND C. J. K. BATTY
}

\begin{abstract}
The main result is the following stability theorem: Let $\tau=$ $(T(t))_{t \geq 0}$ be a bounded $C_{0}$-semigroup on a reflexive space $X$. Denote by $A$ the generator of $\tau$ and by $\sigma(A)$ the spectrum of $A$. If $\sigma(A) \cap i \mathbf{R}$ is countable and no eigenvalue of $A$ lies on the imaginary axis, then $\lim _{t \rightarrow \infty} T(t) x=0$ for all $x \in X$.
\end{abstract}

1. Introduction. The asymptotic behavior of solutions of a differential equation is frequently related to spectral properties of the underlying operator. This is well illustrated by the following classical theorem due to Liapunov.

Let $A$ be an $n \times n$-matrix. Then $\lim _{t \rightarrow \infty} u(t)=0$ for every solution $u$ of the differential equation

$$
u^{\prime}(t)=A u(t) \quad(t \geq 0)
$$

if and only if the spectrum of $A$ lies in the open left half plane.

In this paper we discuss generalizations of this theorem to infinite dimensions.

Let $\tau=(T(t))_{t>0}$ be a $C_{0}$-semigroup on a Banach space $X$. We say $\tau$ is stable if $\lim _{t \rightarrow \infty} T(t) x=0$ for all $x \in X$. This means that the generalized solutions of the differential equation $u^{\prime}(t)=A u(t)(t \geq 0)$ tend to 0 with $t \rightarrow \infty$.

Our aim is to find spectral conditions on $A$ which imply the stability of $\tau$.

There are two features which differ greatly from the finite dimensional case.

1. If $T$ is stable, then $A$ has no eigenvalues on the imaginary axis, but it can happen that $\sigma(A) \cap i \mathbf{R} \neq \varnothing$.

2. The spectral mapping theorem does not hold in general. In particular, it can happen that $\operatorname{Re} \lambda<0$ for all $\lambda \in \sigma(A)$ (or even $\sigma(A)=\varnothing[\mathbf{1 2}]$ ) but $T$ is unbounded (see the discussion in $[8, \mathrm{~A}-\mathrm{III}]$ ).

However, assuming boundedness, our main result is the following (which is easiest to formulate for reflexive spaces).

StABILITy TheOREM. Let $X$ be reflexive. Assume that $\tau$ is bounded and no eigenvalue of $A$ lies on the imaginary axis. If $\sigma(A) \cap i \mathbf{R}$ is countable, then $\tau$ is stable.

This theorem is best possible in the following sense: For any closed uncountable set $E \subset \mathbf{R}$ we give an example of a bounded unstable semigroup on a reflexive space such that $\sigma(A) \subset i E$ and $P \sigma(A)=\varnothing$.

Received by the editors March 16, 1987.

1980 Mathematics Subject Classification (1985 Revision). Primary 47D05; Secondary 47A10, $44 \mathrm{~A} 10$.

Key words and phrases. Tauberian theorems, $C_{0}$-semigroup, stability, power bounded, Laplace transform, residual spectrum. 
In the case when $\sigma(A) \cap i \mathbf{R}$ is empty (even if $X$ is nonreflexive), the stability theorem follows easily from a Tauberian theorem of Ingham [4]. Simple proofs of special cases of Ingham's theorem have been given by Newman [9] for Dirichlet series, and by Korevaar [6] and Zagier [13] for Laplace transforms, each being part of a simple proof of the prime number theorem. Our proof of the stability theorem is based on a refinement of the techniques used by Newman, Korevaar and Zagier.

Whereas they assumed that the Laplace transform is analytic across the imaginary axis, and Ingham required the Laplace transform to be continuously extendible to the imaginary axis, our main effort consists in extending the estimates to the case when the Laplace transform behaves irregularly at some points of the axis.

We also prove the analogous stability theorem for discrete semigroups. The result is related to a Tauberian theorem for power series by Allan, O'Farrell and Ransford [1], which stimulated our interest and by which our attention was drawn to the work of Ingham, Newman, Korevaar and Zagier.

ACKNOWLEDGEMENT. One of the authors (W.A.) would like to express his gratitude for a SERC-grant. He also thanks St. John's College for its hospitality and generosity during his stay in Oxford.

2. The Stability Theorem for semigroups. Throughout this section we denote by $\tau=(T(t))_{t \geq 0}$ a $C_{0}$-semigroup on a Banach space $X$ and by $A$ the generator of $\tau$. If $\tau$ is stable, then $\tau$ is bounded by the uniform boundedness principle. Hence the spectrum of $A$ is contained in the left half-plane $\{\lambda \in \mathrm{C}: \operatorname{Re} \lambda \leq 0\}$.

There is a condition on $\sigma(A) \cap i \mathbf{R}$ which is necessary for stability.

Proposition 2.1. If $\tau$ is stable, then $R \sigma(A) \cap i \mathbf{R}=\varnothing$.

Here we denote by $R \sigma(A)$ the residual spectrum of $A$, this is by definition the set of all $\lambda \in \mathrm{C}$ such that range $(\lambda-A)$ is not dense in $X$; and so, by the Hahn-Banach theorem, $R \sigma(A)=P \sigma\left(A^{\prime}\right)$, the point spectrum of the adjoint $A^{\prime}$ of $A$.

Proof. Assume that there exists $s \in \mathbf{R}$ such that $i s \in R \sigma(A)$. Then there exists $x^{\prime} \in X^{\prime}, x^{\prime} \neq 0$, such that $T(t)^{\prime} x^{\prime}=\exp (i s t) \cdot x^{\prime}(t \geq 0)$. Let $x \in X$ such that $\left\langle x, x^{\prime}\right\rangle=1$. Then $\left\langle T(t) x, x^{\prime}\right\rangle=\exp (i s t)(t \geq 0)$. Hence $\tau$ is not stable.

Usually the condition that $R \sigma(A) \cap i \mathbf{R}=\varnothing$ is easy to check. This is in particular the case when $X$ is reflexive. In fact, then the following holds.

Proposition 2.2. If $X$ is reflexive and $\tau$ is bounded, then $R \sigma(A) \cap i \mathbf{R}=$ $\operatorname{P\sigma }(A) \cap i \mathbf{R}$.

This is a consequence of the following lemma.

LEMMA 2.3. If $T$ is bounded (and $X$ arbitrary), then $\operatorname{P\sigma }(A) \cap i \mathbf{R} \subset R \sigma(A)$.

PROOF OF LEMMA 2.3. Let $\eta \in P \sigma(A) \cap i \mathbf{R}$. We can assume that $\eta=0$ (rescaling the semigroup otherwise). Then there exists $u \in X, u \neq 0$, such that $T(t) u=u(t \geq 0)$. Let $u^{\prime} \in X^{\prime}$ such that $\left\langle u, u^{\prime}\right\rangle=1$. Let $\phi$ be a translationinvariant positive linear form on $L^{\infty}[0, \infty)$ satisfying $\phi(1)=1$, where 1 denotes the constant-1-function. Define $x^{\prime} \in X$ by $\left\langle x, x^{\prime}\right\rangle=\phi\left(\left\langle T(\cdot) x, u^{\prime}\right\rangle\right)$. Then $\left\langle u, x^{\prime}\right\rangle=$ $\phi\left(\left\langle T(\cdot) u, u^{\prime}\right\rangle\right)=\phi(1)=1$, and $\left\langle T(t) x, x^{\prime}\right\rangle=\phi\left(\left\langle T(\cdot+t) x, u^{\prime}\right\rangle\right)=\phi\left(\left\langle T(\cdot) x, u^{\prime}\right\rangle\right)=$ $\left\langle x, x^{\prime}\right\rangle$ for all $x \in X$. Hence $x^{\prime} \in D\left(A^{\prime}\right), x^{\prime} \neq 0$, and $A^{\prime} x^{\prime}=0$.

PROOF OF PROPOSITION 2.2. It follows from Lemma 2.3 that $\operatorname{P\sigma }(A) \cap i \mathbf{R} \subset$ $R \sigma(A)$ and (since $A^{\prime}$ is the generator of a bounded semigroup) $R \sigma(A) \cap i \mathbf{R}=$ $P \sigma\left(A^{\prime}\right) \cap i \mathbf{R} \subset R \sigma\left(A^{\prime}\right)=P \sigma\left(A^{\prime \prime}\right)=P \sigma(A)$. 
The following is our main theorem.

STABILITY THEOREM 2.4. Let $T$ be a bounded $C_{0}$-semigroup with generator A. Assume that $R \sigma(A) \cap i \mathbf{R}=\varnothing$. If $\sigma(A) \cap i \mathbf{R}$ is countable, then $\tau$ is stable.

We proceed with a discussion of this result. The proof will be given in the next section.

First we show by several counterexamples that the Stability Theorem is best possible in several respects.

EXAMPLE 2.5. (a) Let $E \subset \mathbf{R}$ be closed and uncountable. Then there exists a unitary group $\mathcal{U}=(U(t))_{t \in \mathbf{R}}$ whose generator $B$ satisfies $\sigma(B) \subset i E, R \sigma(B)=\varnothing$. But $\mathcal{U}$ is not stable (in fact, $\lim _{t \rightarrow \infty} U(t) x=0$ implies $x=0$ ).

ProOF. There exists a nonatomic probability measure $\mu$ on $E$. Let $X=$ $L^{2}(E, \mu)$ and $(U(t) f)(s)=e^{i s t} f(s)(t \in \mathbf{R})$.

(b) The boundedness condition on $T$ cannot be weakened considerably: The growth bound (or type) $\omega(\tau)$ of $\tau$ is defined by $\omega(\tau)=\inf \left\{w \in \mathbf{R}:\|T(t)\| \leq M e^{w t}\right.$ $(t \geq 0)$ for some $M \geq 1\}$.

There exists a $C_{0}$-semigroup $\tau$ such that $\omega(\tau)=0, \operatorname{Re} \lambda<0$ for all $\lambda \in \sigma(A)$, but $T$ is not stable.

PROOF. Let $X=c_{0},(T(t) x)_{2 n-1}=\exp \left(-t / n^{2}+i n t\right) \cdot\left(x_{2 n-1}+t x_{2 n}\right),(T(t) x)_{2 n}$ $=\exp \left(-t / n^{2}+i n t\right) \cdot x_{2 n}$. Then $\omega(\tau)=0$. The generator $A$ is given by $(A x)_{2 n-1}=$ $\left(-1 / n^{2}+i n\right) x_{2 n-1}+x_{2 n},(A x)_{2 n}=\left(-1 / n^{2}+i n\right) x_{2 n}$ whenever this defines an element in $c_{0}$. Hence $\sigma(A) \cap i \mathbf{R}=\varnothing$. Now let $y_{2 n-1}=0, y_{2 n}=1 / n^{2}$. Then $y \in D(A),\|T(t) y\|=\sup _{n \in \mathbf{N}} t / n^{2} \cdot \exp \left(-t / n^{2}\right) \rightarrow e^{-1}$ as $t \rightarrow \infty$. Thus $\tau$ is not stable.

(c) It is not possible to find necessary and sufficient spectral conditions for stability.

In fact, let $X=C_{0}[0, \infty)$ and $(T(t) f)(x)=f(x+t)$. Then $\sigma(A)=\{\lambda \in$ C: $\operatorname{Re} \lambda \leq 0\}$ and $\tau$ is stable.

Next we give a consequence of the Stability Theorem.

COROLlaRY 2.6. Let $X$ be reflexive and $\tau=(T(t))_{t \geq 0}$ be a bounded $C_{0}$ semigroup with generator $A$. If $\sigma(A) \cap i \mathbf{R}$ is countable, then $\bar{X}$ is the direct sum of the invariant closed subspaces $X_{s}$ and $X_{g}$, where $X_{s}=\left\{x \in X: \lim _{t \rightarrow \infty} T(t) x=0\right\}$ and $X_{g}=\overline{\operatorname{span}}\{x \in D(A): A x=\lambda x$ for some $\lambda \in i \mathbf{R}\}$.

Moreover, the restriction of $\tau$ to $X_{g}$ can be extended to a bounded $C_{0}$-group on $X_{g}$.

PROOF. By the splitting theorem of Jacobs, Deleeuw and Glicksberg [7, Theorem 4.4, p. 105] $X$ is the direct sum of $X_{g}$ and a closed subspace $X_{s}$ which both are invariant under $\tau$. In addition, denoting by $\tau_{g}$ and $\tau_{s}$ the restriction semigroup of $\tau$ to $X_{g}$ and $X_{s}$, respectively, and by $A_{g}$ and $A_{s}$ their generators, then the closure of $\tau_{g}$ with respect to the weak operator topology is a multiplicative group of operators on $X_{g}$. Hence $\tau_{g}$ consists of bijective operators and consequently, $\tau_{g}$ can be extended to a bounded $C_{0}$-group on $X_{g}$.

Moreover, $\operatorname{P\sigma }\left(A_{s}\right) \cap i \mathbf{R}=\varnothing$ by construction. Since $\sigma\left(A_{s}\right) \subset \sigma(A)$, it follows from the Stability Theorem 2.4 in conjunction with Proposition 2.2 that $\tau_{s}$ is stable.

In conclusion, we mention a case where the assumption that $\tau$ is a priori bounded can be omitted. 
Proposition 2.7. Assume that $T$ is eventually norm continuous (i.e. there exists $t_{0} \geq 0$ such that $\left.\lim _{h \rightarrow \infty}\left\|T\left(t_{0}+h\right)-T\left(t_{0}\right)\right\|=0\right)$.

If $\operatorname{Re} \lambda<0$ for all $\lambda \in \sigma(A)$, then $\tau$ is stable.

Proof. Since $T$ is eventually norm continuous, the set $C:=\{\lambda \in \mathbf{C}: \operatorname{Re} \lambda \geq$ $-1\}$ is compact (see e.g. [8, A-II Theorem 1.20]). Consequently, $s(A):=\sup \{\operatorname{Re} \lambda$ : $\lambda \in \sigma(A)\}<0$. Hence by [8, A-III 6.6], $\omega(\tau)<0$, and so $\lim _{t \rightarrow \infty}\|T(t)\|=0$.

3. Proof of the Stability Theorem. The following is our main estimate for Laplace transforms. It is here where we use Newman's [9] technique (see also Korevaar [6] and Zagier [13]). Analogous estimates for power series are given by Allan, O'Farrell and Ransford [1].

LEMMA 3.1. Let $X$ be a Banach space and $f:[0, \infty) \rightarrow X$ be a bounded, strongly measurable function. Denote by

$$
g(z)=\int_{0}^{\infty} e^{-z t} f(t) d t \quad(\operatorname{Re} z>0)
$$

its Laplace transform. Let $i E$ be the set of all singular points of $g$ on the imaginary axis. Suppose that $0 \notin E$. Let $R>0, \xi_{j} \in \mathbf{R}, 0<\varepsilon_{j}<\left|\xi_{j}\right|(j=1, \ldots, n)$ such that the intervals $(-\infty,-R),(R, \infty),\left(\xi_{j}-\varepsilon_{j}, \xi_{j}+\varepsilon_{j}\right)(j=1, \ldots, n)$ are disjoint and cover $E$. Suppose further that for $j=1, \ldots, n$ there exist $\eta_{j} \in\left(\xi_{j}-\varepsilon_{j}, \xi_{j}+\varepsilon_{j}\right)$ such that

$$
M_{j}:=\sup _{t \geq 0}\left\|\int_{0}^{t} \exp \left(-i \eta_{j} s\right) f(s) d s\right\|<\infty \quad(j=1, \ldots, n) .
$$

Then

$$
\begin{aligned}
& \limsup _{t \rightarrow \infty}\left\|\int_{0}^{t} f(s) d s-g(0)\right\| \\
& \quad \leq \frac{2 M_{0}}{R} \prod_{j=1}^{n} a_{j}+12 \sum_{j=1}^{n} M_{j} \varepsilon_{j} \xi_{j}^{2}\left(\left|\xi_{j}\right|-\varepsilon_{j}\right)^{-1}\left(\xi_{j}^{2}-\varepsilon_{j}^{2}\right)^{-1} \prod_{k=1, k \neq j}^{n} b_{j k}
\end{aligned}
$$

where $M_{0}=\sup _{t \geq 0}\|f(t)\|$

$$
\begin{aligned}
a_{j} & =\left(1+\varepsilon_{j}^{2}\left(R-\left|\xi_{j}\right|\right)^{-2}\right) \xi_{j}^{2}\left(\xi_{j}^{2}-\varepsilon_{j}^{2}\right)^{-1}, \\
b_{j k} & =\left(1+\varepsilon_{k}^{2}\left(\left|\xi_{j}-\xi_{k}\right|-\varepsilon_{j}\right)^{-2}\right) \xi_{k}^{2}\left(\xi_{k}^{2}-\varepsilon_{k}^{2}\right)^{-1} \quad(k \neq j) .
\end{aligned}
$$

PROOF. After renumbering, we can arrange that

$$
-R \leq \xi_{1}-\varepsilon_{1}<\xi_{1}+\varepsilon_{1} \leq \xi_{2}-\varepsilon_{2}<\xi_{2}+\varepsilon_{2} \leq \xi_{3}-\varepsilon_{3}<\cdots<\xi_{n}+\varepsilon_{n} \leq R .
$$

Consider $g$ extended to a holomorphic function in a simply-connected open set $U$ containing $\{z$ : $\operatorname{Re} z \geq 0, z \notin i E\}$, and take a contour $\gamma$ in $U$ consisting of the righthand half of the circle $|z|=R$, the right-hand halves of the circles $\left|z-i \xi_{j}\right|=\varepsilon_{j}$ and smooth paths $\gamma_{j}(0 \leq j \leq n)$ joining $-i R$ to $i\left(\xi_{1}-\varepsilon_{1}\right)(j=0), i\left(\xi_{j}+\varepsilon_{j}\right)$ to $i\left(\xi_{j+1}-\varepsilon_{j+1}\right)(0<j<n)$ and $i\left(\xi_{n}+\varepsilon_{n}\right)$ to $i R(j=n)$ lying entirely (except at the endpoints) within $U \cap(\operatorname{Re} z<0\}$. Then $\gamma$ is a closed contour, which may be taken to be simple, with 0 in its interior. 
Let

$$
\begin{aligned}
h_{j}(z) & =\left(1+\varepsilon_{j}^{2}\left(z-i \xi_{j}\right)^{-2}\right) \xi_{j}^{2}\left(\xi_{j}^{2}-\varepsilon_{j}^{2}\right)^{-1}, \\
h(z) & =\left(1+z^{2} / R^{2}\right) \prod_{j=1}^{n} h_{j}(z), \\
g_{t}(z) & =\int_{0}^{t} e^{-s z} f(s) d s \quad(z \in \mathbf{C}, t \geq 0) .
\end{aligned}
$$

By Cauchy's theorem,

$$
\left(g(0)-g_{t}(0)\right)=\frac{1}{2 \pi i} \int_{\gamma} h(z)\left(g(z)-g_{t}(z)\right) e^{t z} z^{-1} d z .
$$

We estimate the integral on the different parts of $\gamma$.

(a) On $|z|=R, \operatorname{Re} z>0$. If $z=R e^{i \theta}(-\pi / 2<\theta<\pi / 2)$, then

$$
\begin{aligned}
\left\|\left(g(z)-g_{t}(z)\right) e^{t z}\right\|= & \left\|\int_{t}^{\infty} e^{-(s-t) z} f(s) d s\right\|=\left\|\int_{0}^{\infty} e^{-r z} f(r+t) d r\right\| \\
& \leq M_{0} \int_{0}^{\infty} e^{-r \operatorname{Re} z} d r=M_{0}(R \cos \theta)^{-1},
\end{aligned}
$$

$\left|1+z^{2} / R^{2}\right|=2 \cos \theta,\left|h_{j}(z)\right| \leq a_{j}$. Hence

$$
\left\|\int_{|z|=R ; \operatorname{Re} z>0} h(z)\left(g(z)-g_{t}(z)\right) e^{t z} z^{-1} d z\right\| \leq \frac{2 M_{0} \pi}{R} \prod_{j=1}^{n} a_{j} .
$$

(b) We consider the integral on $\left|z-i \xi_{j}\right|=\varepsilon_{j}$. If $z=i \xi_{j}+\varepsilon_{j} e^{i \theta}(-\pi / 2<\theta<\pi / 2)$, then letting $F_{j}(t)=\int_{0}^{t} \exp \left(-i \eta_{j} s\right) f(s) d s$ we obtain $\left\|\left(g(z)-g_{t}(z)\right) e^{t z}\right\|$

$$
\begin{aligned}
& =\left\|e^{t z} \int_{t}^{\infty} \exp \left(-s\left(i\left(\xi_{j}-\eta_{j}\right)+\varepsilon_{j} e^{i \theta}\right)\right) \exp \left(-i \eta_{j} s\right) f(s) d s\right\| \\
& =\| e^{t z}\left\{-\exp \left(-t\left(i\left(\xi_{j}-\eta_{j}\right)+\varepsilon_{j} e^{i \theta}\right)\right) \cdot F_{j}(t)\right. \\
& \left.\quad+\left(i\left(\xi_{j}-\eta_{j}\right)+\varepsilon_{j} e^{i \theta}\right) \int_{t}^{\infty} \exp \left(-s\left(i\left(\xi_{j}-\eta_{j}\right)+\varepsilon_{j} e^{i \theta}\right)\right) F_{j}(s) d s\right\} \| \\
& \leq M_{j}+2 \varepsilon_{j} M_{j} \int_{t}^{\infty} e^{-(s-t) \varepsilon_{j} \cos \theta} d s \\
& =M_{j}(1+2 / \cos \theta) \leq 3 M_{j} / \cos \theta, \\
& \quad\left|1+z^{2} / R^{2}\right| \leq 2, \quad\left|h_{j}(z)\right|=2 \cos \theta \cdot \xi_{j}^{2}\left(\xi_{j}^{2}-\varepsilon_{j}^{2}\right)^{-1}, \\
& \quad\left|h_{k}(z)\right| \leq b_{j k} \quad(k \neq j), \quad\left|z^{-1}\right| \leq\left(\left|\xi_{j}\right|-\varepsilon_{j}\right)^{-1} .
\end{aligned}
$$

Hence

$$
\begin{gathered}
\left\|\int_{\left|z-i \xi_{j}\right|=\varepsilon_{j} ; \operatorname{Re} z>0} h(z)\left(g(z)-g_{t}(z)\right) e^{t z} z^{-1} d z\right\| \\
\leq \varepsilon_{j} 12 M_{j} \pi \xi_{j}^{2}\left(\left|\xi_{j}\right|-\varepsilon_{j}\right)^{-1}\left(\xi_{j}^{2}-\varepsilon_{j}^{2}\right)^{-1} \prod_{\substack{k=1 \\
k \neq j}}^{n} b_{j k} .
\end{gathered}
$$


(c) By the bounded convergence theorem,

$$
\lim _{t \rightarrow \infty} \int_{\gamma_{j}} h(z) g(z) e^{t z} z^{-1} d z=0 .
$$

(d) Since $g_{t}$ is an entire function,

$$
\begin{array}{rl}
\int_{\gamma_{0} \cup \cdots \cup \gamma_{n}} & h(z) g_{t}(z) e^{t z} z^{-1} d z \\
= & \int_{|z|=R ; \operatorname{Re} z<0} h(z) g_{t}(z) e^{t z} z^{-1} d z \\
& +\sum_{j=1}^{n} \int_{\left|z-i \xi_{j}\right|=\varepsilon_{j} ; \operatorname{Re} z<0} h(z) g_{t}(z) e^{t z} z^{-1} d z .
\end{array}
$$

If $z=R e^{i \theta}(\pi / 2<\theta<3 \pi / 2)$ then

$$
\begin{aligned}
\left\|g_{t}(z) e^{t z}\right\| & =\left\|\int_{0}^{t} e^{-(s-t) z} f(s) d s\right\| \leq M_{0} \int_{0}^{t} e^{-(s-t) R \cos \theta} d s \\
& \leq M_{0}(R|\cos \theta|)^{-1} .
\end{aligned}
$$

So estimating as in (a) we obtain

$$
\left\|\int_{|z|=R ; \operatorname{Re} z<0} h(z) g_{t}(z) e^{t z} z^{-1} d z\right\| \leq \frac{2 M_{0} \pi}{R} \prod_{j=1}^{n} a_{j} .
$$

For $z=i \xi_{j}+\varepsilon_{j} e^{i \theta}(\pi / 2<\theta<3 \pi / 2)$ we have

$$
\begin{aligned}
\left\|g_{t}(z) e^{t z}\right\|= & \left\|e^{t z} \int_{0}^{t} \exp \left(-s\left(i\left(\xi_{j}-\eta_{j}\right)+\varepsilon_{j} e^{i \theta}\right)\right) \exp \left(-i \eta_{j} s\right) f(s) d s\right\| \\
= & \| \exp \left(i t \eta_{j}\right) F_{j}(t)+\left(i\left(\xi_{j}-\eta_{j}\right)+\varepsilon_{j} e^{i \theta}\right) \\
& \cdot \int_{0}^{t} \exp \left(t z-s\left(i\left(\xi_{j}-\eta_{j}\right)+\varepsilon_{j} e^{i \theta}\right)\right) F_{j}(s) d s \| \\
\leq & M_{j}+2 \varepsilon_{j} M_{j} \int_{0}^{t} e^{-(s-t) \varepsilon_{j} \cos \theta} d s \\
\leq & 3 M_{j} /|\cos \theta| .
\end{aligned}
$$

Estimating as in (b) we obtain

$$
\begin{aligned}
& \left\|\int_{\left|z-i \xi_{j}\right|=\varepsilon_{j} ; \operatorname{Re} z<0} h(z) g_{t}(z) e^{t z} z^{-1} d z\right\| \\
& \quad \leq \varepsilon_{j} \cdot 12 M_{j} \cdot \pi \cdot \xi_{j}^{2} \cdot\left(\left|\xi_{j}\right|-\varepsilon_{j}\right)^{-1}\left(\xi_{j}^{2}-\varepsilon_{j}^{2}\right)^{-1} \prod_{\substack{k=1 \\
k \neq j}}^{n} b_{j k} .
\end{aligned}
$$

Now (3.1) follows from (3.2)-(3.8).

REMARK 3.2. As a particular case of Lemma 3.1 we obtain: If $E \cap[-R, R]=\varnothing$, then

$$
\limsup _{t \rightarrow \infty}\left\|\int_{0}^{t} f(s) d s-g(0)\right\| \leq \frac{2 M_{0}}{R}
$$


This is precisely what is proved by Korevaar [6] and Zagier [13].

PROOF OF THE STABILITY ThEOREM. Let $\tau=(T(t))_{t>0}$ be a $C_{0}$-semigroup such that $M:=\sup _{t \geq 0}\|T(t)\|<\infty$. Denote by $A$ the generator of $\tau$ and assume that $R \sigma(A) \cap i \mathbf{R}=\bar{\varnothing}$ and that $E:=\{\eta \in \mathbf{R}: i \eta \in \sigma(A)\}$ is countable. Rescaling $\tau$ if necessary we can assume that $0 \notin E$.

Let $R>0$ such that $\pm R \notin E$ and let $E_{0}=[-R, R] \cap E$. For an ordinal $\alpha>0$ let $E_{\alpha}$ be the set of all cluster points of $E_{\alpha-1}$ if $\alpha$ is nonlimit, and $E_{\alpha}=\bigcap_{\beta<\alpha} E_{\beta}$, if $\alpha$ is a limit ordinal.

We shall prove the following inductive statement.

Let $\alpha$ be an ordinal. If $E_{\alpha}=\varnothing$, then

$$
\limsup _{t \rightarrow \infty}\left\|T(t) A^{-1} x\right\| \leq \frac{2 M}{R}\|x\| \quad(x \in X) ;
$$

if $E_{\alpha}$ is covered by disjoint intervals $\left(\eta_{j}-\varepsilon_{j}, \eta_{j}+\varepsilon_{j}\right)(j=1, \ldots, n)$, where $\eta_{j} \in E_{\alpha}$, $\left|\eta_{j}\right|-\varepsilon_{j}>0, R-\left|\eta_{j}\right|-\varepsilon_{j}>0$ and $\eta_{j} \pm \varepsilon_{j} \notin E(j=1, \ldots, n)$, then

$$
\begin{aligned}
& \limsup _{t \rightarrow \infty}\left\|T(t) A^{-1} U x\right\| \leq \frac{2 M\|U x\|}{R} \prod_{j=1}^{n} \alpha_{j} \\
& \quad+48 \pi M \sum_{j=1}^{n}\left\|U_{j} x\right\|\left|\eta_{j}\right| \varepsilon_{j}\left(\left|\eta_{j}\right|-\varepsilon_{j}\right)^{-1}\left(\eta_{j}^{2}-\varepsilon_{j}^{2}\right)^{-1} \prod_{\substack{k=1 \\
k \neq j}}^{n} \beta_{j k}
\end{aligned}
$$

where

$$
\begin{aligned}
U= & \prod_{j=1}^{n}\left[T\left(\frac{2 \pi}{\left|\eta_{j}\right|}\right)-I\right], \\
U_{j}= & \prod_{\substack{k=1 \\
k \neq j}}^{n}\left[T\left(\frac{2 \pi}{\left|\eta_{k}\right|}\right)-I\right], \\
\alpha_{j}= & \left(1+\varepsilon_{j}^{2}\left(R-\left|\eta_{j}\right|\right)^{-2}\right) \eta_{j}^{2}\left(\eta_{j}^{2}-\varepsilon_{j}^{2}\right)^{-1}, \\
\beta_{j k}= & \left(1+\varepsilon_{k}^{2}\left(\left|\eta_{j}-\eta_{k}\right|-\varepsilon_{j}\right)^{-2}\right) \eta_{k}^{2}\left(\eta_{k}^{2}-\varepsilon_{k}^{2}\right)^{-1} \quad(k \neq j) .
\end{aligned}
$$

Once this statement has been established, the theorem is proved as follows.

Since $E_{\alpha}$ is compact and countable, $E_{\alpha}$ is either empty or contains isolated points, so that $E_{\alpha}=\varnothing$ or $E_{\alpha+1} \neq E_{\alpha}$. Thus it follows that for some $\alpha$ (at most $\left.\omega_{1}\right), E_{\alpha}=\varnothing$. Hence by the inductive statement, (3.10) holds. Since $R>0$ can be chosen arbitrarily large, it follows that $\lim _{t \rightarrow \infty} T(t) x=0$ for all $x \in D(A)$. Since $D(A)$ is dense in $X$ and $\tau$ is bounded, this implies that $\tau$ is stable.

Thus it remains to prove the inductive statement. First, consider the case $\alpha=0$. Take $x$ in $X$, and put $f(t)=T(t) U x(t \geq 0)$. Then

$$
g(z):=\int_{0}^{\infty} e^{-t z} T(t) U x d t=R(z, A) U x \quad(\operatorname{Re} z>0) .
$$

Thus the singular set of $g$ on $i \mathbf{R}$ is contained in $i E$ and $g(0)=-A^{-1} U x$. Furthermore,

$$
\int_{0}^{t} f(s) d s=\int_{0}^{t} T(s) A A^{-1} U x d s=T(t) A^{-1} U x-A^{-1} U x .
$$


Hence $\left\|T(t) A^{-1} U x\right\|=\left\|\int_{0}^{t} f(s) d s-g(0)\right\|$. Moreover, $\|f(t)\| \leq M\|U x\|(t \geq 0)$. So letting $U=I$ the assertion follows from Remark 3.2 in the case when $E_{0}=\varnothing$. In the other case we have

$$
\begin{aligned}
\int_{0}^{t} \exp \left(-i \eta_{j} s\right) f(s) d s= & \int_{0}^{t} \exp \left(-i \eta_{j} s\right) T(s)\left[T\left(\frac{2 \pi}{\left|\eta_{j}\right|}\right)-I\right] \dot{U}_{j} x d s \\
= & \int_{2 \pi /\left|\eta_{j}\right|}^{t+2 \pi /\left|\eta_{j}\right|} \exp \left(-i \eta_{j} s\right) T(s) U_{j} x d s \\
& -\int_{0}^{t} \exp \left(-i \eta_{j} s\right) T(s) U_{j} x d s \\
= & \int_{t}^{t+2 \pi /\left|\eta_{j}\right|} \exp \left(-i \eta_{j} s\right) T(s) U_{j} x d s \\
& -\int_{0}^{2 \pi /\left|\eta_{j}\right|} \exp \left(-i \eta_{j} s\right) T(s) U_{j} x d s,
\end{aligned}
$$

and so

$$
\left\|\int_{0}^{t} \exp \left(-i \eta_{j} s\right) f(s) d s\right\| \leq \frac{4 \pi}{\left|\eta_{j}\right|} M\left\|U_{j} x\right\| \quad(j=1, \ldots, n) .
$$

Thus (3.11) follows from Lemma 3.1.

Now suppose that $\alpha$ is an ordinal $>0$ such that the statement is true for all ordinals $\beta<\alpha$. We show that the statement holds for $\alpha$.

First case. $\alpha$ is a limit ordinal. Then $E_{\alpha}=\bigcap_{\beta<\alpha} E_{\beta}$. If $E_{\alpha}=\varnothing$, then (by compactness) there exists $\beta<\alpha$ such that $E_{\beta}=\varnothing$. So (3.10) follows from the inductive assumption. If $E_{\alpha}$ is contained in the union of $\left(\eta_{j}-\varepsilon_{j}, \eta_{j}+\varepsilon_{j}\right)$ $(j=1, \ldots, n)$ according to the statement, then (by compactness) there exists $\beta<\alpha$ such that $E_{\beta}$ is contained in this union. So the inductive hypothesis yields (3.11).

Second case. $\alpha$ is a nonlimit ordinal. Suppose that $E_{\alpha} \subset \bigcup_{j=1}^{n}\left(\eta_{j}-\varepsilon_{j}, \eta_{j}+\varepsilon_{j}\right)$ according to the statement. Then there are only finitely many points $\eta_{n+1}, \ldots$, $\eta_{n+p} \in E_{\alpha-1}$ which do not lie in any of these intervals. Take $\varepsilon_{j}>0(j=$ $n+1, \ldots, n+p)$ such that the intervals $\left(\eta_{j}-\varepsilon_{j}, \eta_{j}+\varepsilon_{j}\right)(j=1, \ldots, n+p)$ are disjoint and such that $\eta_{j} \pm \varepsilon_{j} \notin E,\left|\eta_{j}\right|-\varepsilon_{j}>0, R>\left|\eta_{j}\right|+\varepsilon_{j}(j=n+1, \ldots, n+p)$.

Then $E_{\alpha-1} \subset \bigcup_{j=1}^{n+p}\left(\eta_{j}-\varepsilon_{j}, \eta_{j}+\varepsilon_{j}\right)$. Let

$$
\begin{aligned}
V & =\prod_{j=1}^{n+p}\left[T\left(\frac{2 \pi}{\left|\eta_{j}\right|}\right)-I\right], \\
V_{j} & =\prod_{\substack{k=1 \\
k \neq j}}^{n+p}\left[T\left(\frac{2 \pi}{\left|\eta_{j}\right|}\right)-I\right] \quad(j=1, \ldots, n+p) .
\end{aligned}
$$

Then by the inductive assumption,

$$
\begin{aligned}
\limsup _{t \rightarrow \infty}\left\|T(t) A^{-1} V y\right\| \leq & \frac{2 M\|V y\|}{R} \prod_{j=1}^{n+p} \alpha_{j} \\
& +48 \pi M \sum_{j=1}^{n+p}\left\|V_{j} y\right\|\left|\eta_{j}\right| \varepsilon_{j}\left(\left|\eta_{j}\right|-\varepsilon_{j}\right)^{-1}\left(\eta_{j}^{2}-\varepsilon_{j}^{2}\right)^{-1} \prod_{\substack{k=1 \\
k \neq j}}^{n+p} \beta_{j k}
\end{aligned}
$$


for all $y \in X$. This is true for arbitrarily small $\varepsilon_{j}(j=n+1, \ldots, n+p)$. As $\varepsilon_{j} \rightarrow 0$ $(j=n+1, \ldots, n+p)$, one has

$$
\begin{aligned}
\alpha_{j} & \rightarrow 1 \quad(j=n+1, \ldots, n+p), \\
\beta_{j k} & \rightarrow 1 \quad(k=n+1, \ldots, n+p, j=1, \ldots, n+p, k \neq j), \\
\beta_{j k} & \rightarrow\left(1+\varepsilon_{k}^{2}\left|\eta_{j}-\eta_{k}\right|^{-2}\right) \eta_{k}^{2}\left(\eta_{k}^{2}-\varepsilon_{k}^{2}\right)^{-1} \\
& (k=1, \ldots, n, j=n+1, \ldots, n+p, k \neq j) .
\end{aligned}
$$

Hence for all $y \in X$,

$$
\begin{aligned}
& \limsup _{t \rightarrow \infty}\left\|T(t) A^{-1} V y\right\| \leq \frac{2 M\|V y\|}{R} \prod_{j=1}^{n} \alpha_{j} \\
& \quad+48 \pi M \sum_{j=1}^{n}\left\|V_{j} y\right\|\left|\eta_{j}\right| \varepsilon_{j}\left(\left|\eta_{j}\right|-\varepsilon_{j}\right)^{-1}\left(\eta_{j}^{2}-\varepsilon_{j}^{2}\right)^{-1} \prod_{\substack{k=1 \\
k \neq j}}^{n} \beta_{j k} .
\end{aligned}
$$

Now put $W=\prod_{j=n+1}^{n+p}\left[T\left(2 \pi /\left|\pi_{j}\right|\right)-I\right]$, so that $V=U W, V_{j}=U_{j} W$. Since

$$
1 \notin\left\{\exp \left(2 \pi z /\left|\eta_{j}\right|\right): z \in R \sigma(A)\right\}=R \sigma\left(T\left(2 \pi /\left|\eta_{j}\right|\right)\right) \backslash\{0\}
$$

(observe that the spectral mapping theorem holds for the residual spectrum, see [8, A-III6.3]), each of the operators $\left[T\left(2 \pi /\left|\pi_{j}\right|\right)-I\right]$ has dense range, and so $W$ has dense range. So for $x \in X$, there exists a sequence $\left(y_{r}\right)$ in $X$ such that $\lim _{r \rightarrow \infty} W y_{r}=x$. Applying (3.12) to $y_{r}$, taking the limit for $r \rightarrow \infty$ and using the fact that $\left\|T(t) A^{-1}\right\|$ is bounded, we obtain (3.11).

We obtain (3.10) in the same way in the case when $E_{\alpha}=\varnothing$.

REMARK 3.3 . Let $T=(T(t))_{t \geq 0}$ be a $C_{0}$-semigroup with generator $A$ such that Re $\lambda \leq 0$ for all $\lambda \in \sigma(A), R \sigma(A) \cap i \mathbf{R}=\varnothing$ and $\sigma(A) \cap i \mathbf{R}$ is countable. If instead of boundedness of $\tau$ we assume that there exists a bounded operator $B$ commuting with $T(t)$ for all $t \geq 0$ such that $\sup _{t \geq 0}\|T(t) B\|<\infty$, then $\lim _{t \rightarrow \infty}\|T(t) B x\|=0$ for all $x \in X$.

This is proved by a slight modification of the above. (In the inductive statement we have to write $\limsup _{t \rightarrow \infty}\left\|T(t) A^{-1} U B x\right\|$ on the left-hand side of (3.11) and $\lim \sup _{t \rightarrow \infty}\left\|T(t) A^{-1} B x\right\| \leq 2 M\|x\| / R$ instead of (3.10).)

As a consequence we obtain the following: If $\sup _{t \geq 0}\|T(t) x\|<\infty$ for all $x \in$ $D(A), \sigma(A) \cap i \mathbf{R}$ is countable, $R \sigma(A) \cap i \mathbf{R}=\varnothing$ and $\operatorname{Re} \lambda \leq 0$ for all $\lambda \in \sigma(A)$, then $\lim _{t \rightarrow \infty}\|T(t) x\|=0$ for all $x \in D(A)$. This can be seen by taking $B=R(\lambda, A)$ for some $\lambda \notin \sigma(A)$ in the above statement.

REMARK 3.4 (an "individual stability result"). Let $\tau=(T(t))_{t \geq 0}$ be a $C_{0}$ semigroup with generator $A$. Assume that $\operatorname{Re} \lambda<0$ for all $\lambda \in \sigma(A)$. If $x \in D(A)$ such that $\sup _{t \geq 0}\|T(t) A x\|<\infty$, then $\lim _{t \rightarrow \infty}\|T(t) x\|=0$. Then

PROOF. Let $f(t)=T(t) A x, g(z)=\int_{0}^{\infty} e^{-z t} f(t) d t=R(z, A) A x(\operatorname{Re} z>0)$.

$$
T(t) x=x+\int_{0}^{t} T(s) A x d s=-g(0)+\int_{0}^{t} f(s) d s .
$$

So the claim follows from Remark 3.2 (i.e., from Ingham's Tauberian theorem).

Example 2.5(b) shows that this result is not true if merely $\|T(t) x\|$ is bounded. We do not know whether this "individual stability result" can be extended to the case when $\sigma(A) \cap i \mathbf{R}$ is countable and $\sigma(A) \subset\{\lambda \in \mathbf{C}: \operatorname{Re} \lambda \leq 0\}, R \sigma(A) \cap i \mathbf{R}=\varnothing$. 
4. Two Tauberian theorems for Laplace transforms. Let $f:[0, \infty) \rightarrow X$ be a bounded strongly measurable function with Laplace transform

$$
g(z)=\int_{0}^{\infty} e^{-z t} f(t) d t \quad(\operatorname{Re} z>0)
$$

If $\int_{0}^{t} f(s) d s$ converges for $t \rightarrow \infty$, then it is easy to see that $g(z)$ converges for $z \in \mathbf{R}, z \downarrow 0$ (and the limits coincide). The converse is only true under additional assumptions and corresponding converse results are usually refered to as Tauberian theorems (see, e.g., Widder [11, Chapter 8]). Frequently, these conditions concern the determining function $f$ (a well-known sufficient condition is $\sup _{t \geq 0}\|t f(t)\|<\infty$ to give an example). In Ingham's Tauberian theorem [4], however, a condition on the generating function $g$ is given (namely $(g(z)-g(0)) / z$ should extend continuously to the closed right half-plane)). We give other versions of this theorem in which the set $i E$ of all singularities of $g$ on $i \mathbf{R}$ is a null set and either $g$ is bounded on every bounded subset of the open right half-plane (Theorem 4.4) or

$$
\sup _{\eta \in E} \sup _{t \geq 0}\left\|\int_{0}^{t} e^{-i \eta s} f(s) d s\right\|<\infty
$$

(Theorem 4.1). The power series version of the latter theorem is due to Allan, O'Farrell and Ransford [1] (see also §5).

THEOREM 4.1. Denote by $i E$ the set of all singularities of $g$ on $i \mathbf{R}$. Assume that $E$ is null, $0 \notin E$ and

$$
M:=\sup _{t>0} \sup _{\eta \in E}\left\|\int_{0}^{t} e^{-i \eta s} f(s) d s\right\|<\infty .
$$

Then $\lim _{t \rightarrow \infty} \int_{0}^{t} f(s) d s=g(0)$.

For the proof we shall use the following lemma.

LEMMA 4.2. Let $E$ be a compact null set in $\mathbf{R}$. Then for all $\varepsilon>0$ there exist $\xi_{1}, \ldots, \xi_{n} \in \mathbf{R}$ and $\theta \in(0, \varepsilon / n)$ such that the intervals $\left(\xi_{j}-\theta, \xi_{j}+\theta\right)(j=1, \ldots, n)$ are disjoint and cover $E$.

PROOF. Let $\varepsilon>0$. Since $E$ is a compact null set, there exist open intervals $I_{j}$ $(j=1, \ldots, m)$ which cover $E$ such that $\sum_{j=1}^{m}\left|I_{j}\right|<\varepsilon$. Let $0<\theta<\varepsilon / 4 m$.

The set $F:=\{c \in \mathbf{R}: c+k 2 \theta \in E$ for some $k \in \mathbf{Z}\}$ is a null set. Choose $c \in \mathbf{R} \backslash F$. Let $K=\{k \in \mathbf{Z}:(c+k 2 \theta, c+(k+1) 2 \theta) \cap E \neq \varnothing\}$. Then the intervals $(c+k 2 \theta, c+(k+1) 2 \theta)(k \in K)$ cover $E$ and are pairwise disjoint. Let $K_{1}=\left\{k \in K:(c+k 2 \theta, c+(k+1) 2 \theta) \subset \bigcup_{j=1}^{m} I_{j}\right\}, K_{2}:=K \backslash K_{1}$. Since $K_{2}$ has at most $2 m$ elements these intervals have total length $\operatorname{card}(K) \cdot 2 \theta \leq \varepsilon+2 m 2 \theta<2 \varepsilon$; i.e., $\theta<\varepsilon / \operatorname{card}(K)$ as required.

Proof OF THEOREM 4.1. Let $R>0$ such that $\pm R \notin E$. Let $\delta>0$ such that $|\xi| \geq 2 \delta, R-|\xi| \geq 2 \delta$ for all $\xi \in E \cap[-R, R]$. Let $\varepsilon \in(0, \delta / 2)$. By Lemma 4.2 there exist $\xi_{1}, \ldots, \xi_{n} \in \mathbf{R}, \theta \in(0, \varepsilon / n)$ such that the intervals $\left(\xi_{j}-\theta, \xi_{j}+\theta\right)$ $(j=1, \ldots, n)$ are pairwise disjoint and cover $E \cap[-R, R]$. We may assume that $\left(\xi_{j}-\theta, \xi_{j}+\theta\right) \cap E \neq \varnothing(j=1, \ldots, n)$. Then $\left|\xi_{j}\right| \geq \delta$ and $R-\left|\xi_{j}\right| \geq \delta(j=1, \ldots, n)$. 
We apply Lemma 3.1 using the notation there. Since

$$
\begin{aligned}
& \xi_{j}^{2} \cdot\left(\xi_{j}^{2}-\theta^{2}\right)^{-1}=\left(1-\theta^{2} / \xi_{j}^{2}\right)^{-1} \leq\left(1-\theta^{2} / \delta^{2}\right)^{-1} \\
& \quad \leq 1+2 \theta^{2} / \delta^{2} \leq 1+\theta / \delta \leq e^{\theta / \delta}
\end{aligned}
$$

(note that $\theta / \delta \leq 1 / 2)$ and $1+\theta^{2}\left(R-\left|\xi_{j}\right|\right)^{-2} \leq 1+\theta^{2} / \delta^{2} \leq e^{\theta / \delta}$, it follows that $a_{j} \leq e^{2 \theta / \delta}(j=1, \ldots, n)$ and so $\prod_{j=1}^{n} a_{j} \leq e^{2 n \theta / \delta} \leq e^{2 \varepsilon / \delta} \leq e$.

Moreover, assuming without loss of generality that $\xi_{1}<\xi_{2}<\cdots<\xi_{n}$ we have

$$
\begin{array}{ll}
\xi_{j+r}-\xi_{j}-\theta \geq(2 r-1) \theta & \text { for } r \in\{1, \ldots, n-j\} \text { and } \\
\xi_{j}-\xi_{j-r}-\theta \geq(2 r-1) \theta & \text { for } r \in\{1, \ldots, j-1\} .
\end{array}
$$

Hence

$$
\prod_{\substack{k=1 \\ k \neq j}}^{n} b_{j k}=\prod_{\substack{k=1 \\ k \neq j}}^{n}\left(1+\theta^{2}\left(\left|\xi_{j}-\xi_{k}\right|-\theta\right)^{-2}\right) \xi_{k}^{2}\left(\xi_{k}^{2}-\theta^{2}\right)^{-1} \leq c \cdot e^{(n-1) \theta / \delta}
$$

where $c:=\prod_{r=1}^{\infty}\left(1+(2 r-1)^{-2}\right)^{2}$. So we obtain from $(3.1)$

$$
\begin{aligned}
\limsup _{t \rightarrow \infty} & \left\|\int_{0}^{t} f(s) d s-g(0)\right\| \\
& \leq 2 M_{0} / R \cdot e+12 M n \theta \cdot 2 / \delta \cdot e^{\theta / \delta} \cdot c \cdot e^{(n-1) \theta / \delta} \\
& \leq 2 M_{0} / R \cdot e+\varepsilon \cdot 24 M / \delta \cdot c \cdot e^{\varepsilon / \delta} \\
& \leq 2 M_{0} / R \cdot e+\varepsilon \cdot 24 M / \delta \cdot c \cdot e,
\end{aligned}
$$

where $M_{0}:=\sup _{t \geq 0}\|f(t)\|$. Since $\varepsilon \in(0, \delta / 2)$ was arbitrary, it follows that

$$
\limsup _{t \rightarrow \infty}\left\|\int_{0}^{t} f(s) d s-g(0)\right\| \leq \frac{2 M_{0}}{R} \cdot e,
$$

whenever $\pm R \notin E, R>0$. There exist arbitrarily large $R$ satisfying this, so the claim follows.

In the proof above, we applied Lemma 3.1 with $\varepsilon_{j}=\theta$ for all $j$. So we did not give the best possible estimates. In fact, one can improve (3.1) in such a way that $b_{j k}$ is replaced by $\xi_{k}^{2} /\left(\xi_{k}^{2}-\varepsilon_{k}^{2}\right)$ (cf. (iv) on $[1$, p. 543]). Then $c$ may be replaced by 1 in the estimates above.

EXAMPLE 4.3. The condition (4.1) cannot be omitted in Theorem 4.1. In fact, let $f(t)=\cos t$. Then $g(z)=z /\left(z^{2}+1\right)$. So $E=\{ \pm i\}$, but $\int_{0}^{t} f(s) d s$ does not converge for $t \rightarrow \infty$.

Whereas Theorem 4.1 is based on our main estimate Lemma 3.1, for the next result we use another kind of modification of the Newman-Korevaar-Zagier technique $[9,6,13]$.

THEOREM 4.4. Let $f:[0, \infty) \rightarrow X$ be a bounded strongly measurable function, and let

$$
g(z)=\int_{0}^{\infty} e^{-z t} f(t) d t \quad(\operatorname{Re} z>0)
$$

be its Laplace transform. Assume that $g$ is regular at 0 , that the set $i E$ of all singular points of $g$ on the imaginary axis is a null set, and that for each $y$ in 
$E, g$ is bounded on $\left\{z \in \mathrm{C}: \operatorname{Re} z>0,|z-i y|<\delta_{y}\right\}$ for some $\delta_{y}>0$. Then $\lim _{t \rightarrow \infty} \int_{0}^{t} f(s) d s=g(0)$.

PROOF. Take $R>0$ such that $\pm R \notin E$. By the assumption on $g$, and compactness, there exists $c>0$ such that $\|g(z)\| \leq c$ whenever $\operatorname{Re} z>0,|z| \leq R$. Considering the holomorphic extension of $g$ into the left half-plane, we may find an open set $U$ with the following properties: $\{z \in \mathrm{C}$ : $\operatorname{Re} z \geq 0,|z| \leq R, z \notin i E\} \subset U$, $x+i y \in U, x \leq x^{\prime} \leq 0$ implies $x^{\prime}+i y \in U, g$ is holomorphic on $U,\|g(z)\|<c+1$ for all $z \in U$. There is a continuous path $\gamma_{0}$ of the form

$$
\gamma_{0}(y)=\phi(y)+i y \quad(-R \leq y \leq R)
$$

where $\phi(y) \leq 0(-R \leq y \leq R), \phi(y)=0$ if and only if $y \in\{ \pm R\} \cup E, \gamma_{0}(y) \in U$ if $y \notin\{ \pm R\} \cup E, \phi$ is continuously differentiable on $(-R, R) \backslash E$ such that $\left|\phi^{\prime}(y)\right| \leq 1$ $(y \in(-R, R) \backslash E)$ (observe that $(-R, R) \backslash E$ is the disjoint union of a countable number of open intervals). For $T>0$ put

$$
\begin{aligned}
h_{T}(z) & =e^{T z} \cdot\left(1+z^{2} / R^{2}\right) \cdot 1 / z, \\
J_{T} & =\int_{\gamma} h_{T}(z) g(z) d z,
\end{aligned}
$$

where $\gamma$ is any path in the simply connected region $U \backslash\{x: x \geq 0\}$ from $-i R$ to $i R$. We will show that

$$
\lim _{T \rightarrow \infty}\left\|J_{T}\right\|=0 \text {. }
$$

Admitting (4.2) for a moment, the theorem is proved as follows. Let $g_{T}(z)=$ $\int_{0}^{T} e^{-t z} g(t) d t(T>0)$. Since $g_{T}$ is holomorphic in the entire plane, two applications of Cauchy's theorem show that

$$
\begin{aligned}
g_{T}(0)-g(0)= & \frac{1}{2 \pi i} \int_{|z|=R ; \operatorname{Re} z>0} h_{T}(z)\left(g_{T}(z)-g(z)\right) d z \\
& -\frac{1}{2 \pi i} \int_{\gamma} h_{T}(z)\left(g_{T}(z)-g(z)\right) d z \\
= & \frac{1}{2 \pi i} \int_{|z|=R ; \operatorname{Re} z>0} h_{T}(z)\left(g_{T}(z)-g(z)\right) d z \\
& +\frac{1}{2 \pi i} \int_{|z|=R ; \operatorname{Re} z<0} h_{T}(z) g_{T}(z) d z+\frac{1}{2 \pi i} J_{T} .
\end{aligned}
$$

Now the integrands can be estimated as follows:

$$
\begin{array}{ll}
\left|h_{T}(z)\right|=e^{T \cdot \operatorname{Re} z} \cdot 2|\operatorname{Re} z| / R^{2} & (|z|=R), \\
\left\|g(z)-g_{T}(z)\right\| \leq M \cdot e^{-T \cdot \operatorname{Re} z} / \operatorname{Re} z & (\operatorname{Re} z>0), \\
\left\|g_{T}(z)\right\| \leq M /|\operatorname{Re} z| \cdot e^{-T \cdot \operatorname{Re} z} & (\operatorname{Re} z<0),
\end{array}
$$

where $M:=\sup _{t>0}\|f(t)\|$. Hence $\left\|g(0)-g_{T}(0)\right\| \leq 2 M / R+1 / 2 \pi\left\|J_{T}\right\|$, and so by $(4.2), \lim \sup _{T \rightarrow \infty}\left\|g(0)-g_{T}(0)\right\| \leq 2 M / R$. Since $R$ can be chosen arbitrarily large, it follows that

$$
\lim _{T \rightarrow \infty} \int_{0}^{T} f(s) d s=\lim _{T \rightarrow \infty} g_{T}(0)=g(0) .
$$


It remains to show (4.2). By Cauchy's theorem $J_{T}$ is independent of the choice of the path $\gamma$. In particular, for $0<\varepsilon<-\phi(0) / R^{2}$, taking

$$
\gamma_{\varepsilon}(y)=\phi(y)+\varepsilon\left(R^{2}-y^{2}\right)+i y
$$

we have

$$
J_{T}=\int_{-R}^{R} h_{T}\left(\gamma_{\varepsilon}(y)\right) g\left(\gamma_{\varepsilon}(y)\right)\left(\phi^{\prime}(y)-2 \varepsilon y+i\right) d y .
$$

Letting $\varepsilon \downarrow 0$ and using the bounded convergence theorem, it follows that

$$
J_{T}=\int_{-R}^{R} h_{T}(\gamma(y)) g(\gamma(y))\left(\phi^{\prime}(y)+i\right) d y
$$

(where the integrand is defined a.e.). Now $h_{T}(\gamma(y)) \rightarrow 0(T \rightarrow \infty)$ whenever $y \notin E$. Hence (4.2) follows from the bounded convergence theorem.

5. Stability of discrete semigroups. The analogue of the Stability Theorem 2.4 for power bounded operators holds as well.

THEOREM 5.1. Let $T$ be a bounded operator on $X$ such that

$$
M:=\sup _{n \in \mathbb{N}}\left\|T^{n}\right\|<\infty .
$$

If $\sigma(T) \cap \Gamma$ is countable and $R \sigma(T) \cap \Gamma=\varnothing$, then $\lim _{n \rightarrow \infty} T^{n} x=0$ for all $x \in X$.

Here we denote by $\Gamma:=\{z \in \mathrm{C}:|z|=1\}$ the unit circle.

REMARK 5.2. As in the continuous case, on a reflexive space, the condition $R \sigma(T) \cap \Gamma=\varnothing$ can be replaced by the condition $P \sigma(T) \cap \Gamma=\varnothing$.

The proof is based on the following estimate for power series which is due to Allan, O'Farrell and Ransford [1].

LEMMA 5.3. Let $\left(a_{n}\right)$ be a sequence in $X$ such that the power series $f(z)=$ $\sum_{n=0}^{\infty} a_{n} z^{n}$ has radius of convergence 1 . Let $E$ be the set of all singular points of $f$ in $\Gamma$ and $F=\{\xi \in \mathbf{R}:(\xi-i) /(\xi+i) \in E\}$. Suppose that $1 \notin E$ (so that $F$ is compact and $R_{0}:=\sup \{|z|:(z-i) /(z+i)$ is a singular point of $\left.f\}<\infty\right)$. Then for all $R>R_{0}$ the following holds. If $F$ is contained in the union of disjoint open intervals $\left(\xi_{j}-\varepsilon_{j}, \xi_{j}+\varepsilon_{j}\right)(j=1, \ldots, m)$ where $0<\varepsilon_{j}<1,\left|\xi_{j}\right|+\varepsilon_{j}<R$ such that for some $\eta_{j} \in\left(\xi_{j}-\varepsilon_{j}, \xi_{j}+\varepsilon_{j}\right)$,

$$
M_{j}:=\sup _{N \in \mathbb{N}}\left\|\sum_{n=0}^{N} a_{n}\left(\frac{\eta_{j}-i}{\eta_{j}+i}\right)^{n}\right\|<\infty \quad(j=1, \ldots, m),
$$

then

$$
\begin{aligned}
\underset{N \rightarrow \infty}{\limsup } & \left\|\sum_{n=0}^{N} a_{n}-f(1)\right\| \\
& \leq 16 R^{2} \sum_{j=1}^{m} M_{j} \varepsilon_{j} \prod_{\substack{k=1 \\
k \neq j}}^{m}\left(1+\varepsilon_{k}^{2}\left(\left|\xi_{k}-\xi_{j}\right|-\varepsilon_{j}\right)^{-2}\right) .
\end{aligned}
$$

For the proof we refer to Allan, O'Farrell and Ransford (proof of [1, Theorem $4]$ ). Note that the estimate (iv) on [1, p. 543] is no longer valid, since we do not assume that $\varepsilon_{j} / \varepsilon_{k} \geq 1 / 2$. 
ProOF OF ThEOREM 5.1. Replacing $T$ by $\lambda T$ for some $\lambda \in \Gamma$ we can assume that $1 \notin \sigma(T)$. Let $E=\left\{z \in \Gamma: z^{-1} \in \sigma(T)\right\}, F_{0}=\{\xi \in \mathbf{R}:(\xi-i) /(\xi+i) \in E\}$. For a nonlimit ordinal $\alpha$, let $F_{\alpha}$ be the set of all cluster points of $F_{\alpha-1}$; for a limit ordinal $\alpha$ let $F_{\alpha}=\bigcap_{\beta<\alpha} F_{\beta}$. Fix $R>\sup \{|w|: w \in \mathbf{C},(w+i) /(w-i) \in \sigma(T)\}$. We shall prove by transfinite induction the following statement:

If $F_{\alpha}=\varnothing$, then

$$
\lim _{n \rightarrow \infty}\left\|T^{n} x\right\|=0 \quad(x \in X)
$$

if $F_{\alpha}$ is covered by disjoint subintervals $\left(\xi_{j}-\varepsilon_{j}, \xi_{j}+\varepsilon_{j}\right)$ of $(-R, R)(j=1, \ldots, m)$ such that $\xi_{j} \pm \varepsilon_{j} \notin F_{0}$, then

$$
\begin{gathered}
\limsup _{n \rightarrow \infty}\left\|T^{n} U x\right\| \leq 16(M+1) R^{2} \sum_{j=1}^{m}\left\|U_{j}(I-T) x\right\| \varepsilon_{j} \\
\cdot \sum_{\substack{k=1 \\
k \neq j}}^{m}\left[1+\varepsilon_{k}^{2}\left(\left|\xi_{k}-\xi_{j}\right|-\varepsilon_{j}\right)^{-2}\right]
\end{gathered}
$$

where $U=\prod_{j=1}^{m}\left(\lambda_{j}-T\right), U_{j}=\prod_{\substack{k=1 \\ k \neq j}}^{m}\left(\lambda_{k}-T\right), \lambda_{j}=\left(\xi_{j}+i\right) /\left(\xi_{j}-i\right)(j=1, \ldots, m)$.

Having proved the inductive statement, we argue as follows. For some $\alpha \leq \omega_{1}$, $F_{\alpha}$ is empty. So (5.2) holds, as required.

To prove the inductive statement, first consider the case $\alpha=0$. Take $x \in X$, and put $a_{n}=\left(T^{n}-T^{n+1}\right) U x$. Then

$$
f(z)=\sum_{n=0}^{\infty} z^{n} T^{n}(I-T) U x=(I-z T)^{-1}(I-T) U x \quad(|z|<1) .
$$

Thus the singular set of $f$ is contained in $E$, and $f(1)=U x$,

$$
\sum_{n=0}^{N} a_{n}=\sum_{n=0}^{N}\left(T^{n}-T^{n+1}\right) U x=\left(I-T^{N+1}\right) U x .
$$

Thus $\left\|T^{N+1} U x\right\|=\left\|\sum_{n=0}^{N} a_{n}-f(1)\right\|$. Moreover,

$$
\begin{aligned}
& \sum_{n=0}^{N} a_{n}\left[\frac{\xi_{j}-i}{\xi_{j}+i}\right]^{n}=\sum_{n=0}^{N} a_{n} \lambda_{j}^{-n} \\
& \quad=\sum_{n=0}^{N} \lambda_{j}^{-n} T^{n}\left(\lambda_{j}-T\right)(I-T) U_{j} x \\
& =\lambda_{j}\left(I-\left(\lambda_{j}^{-1} T\right)^{N+1}\right) U_{j}(I-T) x
\end{aligned}
$$

So

$$
\left\|\sum_{n=0}^{N} a_{n}\left[\frac{\xi_{j}-i}{\xi_{j}+i}\right]^{n}\right\| \leq(M+1)\left\|U_{j}(I-T) x\right\| .
$$

Now (5.3) follows from (5.1); and, in the case when $F_{0}=\varnothing$, the spectral radius of $T$ is less than 1 , so (5.2) is trivial.

Now let $\alpha$ be an ordinal $>0$ such that the statement is true for all $\beta<\alpha$. We have to prove that the statement is true for $\alpha$. 
In the case when $\alpha$ is a limit ordinal we argue as in the proof of Theorem 2.4. So assume that $\alpha$ is nonlimit. Assume that $F_{\alpha} \subset \bigcup_{j=1}^{m}\left(\xi_{j}-\varepsilon_{j}, \xi_{j}+\varepsilon_{j}\right)$ according to the inductive statement. There are only finitely many points $\xi_{m+1}, \ldots, \xi_{m+p}$ in $F_{\alpha-1} \backslash \bigcup_{j=1}^{m}\left(\xi_{j}-\varepsilon_{j}, \xi_{j}+\varepsilon_{j}\right)$. Take $\varepsilon_{j} \in(0,1)(j=m+1, \ldots, m+p)$ such that the intervals $\left(\xi_{j}-\varepsilon_{j}, \xi_{j}+\varepsilon_{j}\right)(j=1, \ldots, m+p)$ are disjoint, and $\xi_{j} \pm \varepsilon_{j} \notin F_{0}$. Now $F_{\alpha-1} \subset \bigcup_{j=1}^{m+p}\left(\xi_{j}-\varepsilon_{j}, \xi_{j}+\varepsilon_{j}\right)$. By the inductive hypothesis we obtain for all $y \in X$,

$$
\begin{aligned}
\limsup _{n \rightarrow \infty}\left\|T^{n} V y\right\| \leq & 16(M+1) R^{2} \sum_{j=1}^{m+p}\left\|V_{j}(I-T) y\right\| \varepsilon_{j} \\
& \cdot \sum_{\substack{k=1 \\
k \neq j}}^{m+p}\left[1+\varepsilon_{k}^{2}\left(\left|\xi_{k}-\xi_{j}\right|-\varepsilon_{j}\right)^{-2}\right]
\end{aligned}
$$

where $V=\prod_{j=1}^{m+p}\left(\lambda_{j}-T\right), V_{j}=\prod_{\substack{k=1 \\ k \neq j}}^{m+p}\left(\lambda_{k}-T\right)$.

Letting $\varepsilon_{j} \rightarrow 0(j=m+1, \ldots, m+p)$ we obtain

$$
\begin{gathered}
\limsup _{n \rightarrow \infty}\left\|T^{n} V y\right\| \leq 16(M+1) R^{2} \sum_{j=1}^{m}\left\|V_{j}(I-T) y\right\| \varepsilon_{j} \\
\cdot \sum_{\substack{k=1 \\
k \neq j}}^{m}\left[1+\varepsilon_{k}^{2}\left(\left|\xi_{k}-\xi_{j}\right|-\varepsilon_{j}\right)^{-2}\right] .
\end{gathered}
$$

Now, put $W=\prod_{j=m+1}^{m+p}\left(\lambda_{j}-T\right)$, so that $V=U W, V_{j}=U_{j} W(j=1, \ldots, m)$. Since $\lambda_{j} \notin R \sigma(T), W$ has dense range. So, for any $x \in X$, there exists a sequence $\left(y_{r}\right)$ in $X$ such that $W y_{r} \rightarrow \infty(r \rightarrow \infty)$. Applying (5.4) to $y_{r}$, taking the limit for $r \rightarrow \infty$ and using the fact that $\left\|T^{n}\right\|$ is bounded, (5.3) follows. In the case when $F_{\alpha}=\varnothing,(5.2)$ is proved similarly.

REMARK 5.4. There is a similar result due to Sz.-Nagy-Foias [10, II, Proposition 6.7, p. 85]: If $T$ is a completely nonunitary contraction on a Hilbert space and $\sigma(T) \cap \Gamma$ is null, then $\lim _{n \rightarrow \infty} T^{n} x=0$ for all $x \in X$. The discrete version of Example 2.5(a) shows that this result is no longer true if $T$ is unitary (and $\sigma(T) \cap \Gamma$ uncountable).

The Allan, O'Farrell and Ransford Tauberian theorem for power series $[\mathbf{1}$, Theorem 4] is a generalization of a Tauberian theorem due to Katznelson and Tzafriri [5], which has the following stability result as an easy corollary.

THEOREM 5.6 (KATZNELSON-TZAFRIRI [6]). Let $T$ be a power-bounded operator. Then $\lim _{n \rightarrow \infty}\left\|T^{n+1}-T^{n}\right\|=0$ if and only if $\sigma(T) \cap \Gamma \subset\{1\}$.

In the case when $\sigma(T) \cap \Gamma \subset\{1\}$ and $1 \notin R \sigma(T)$, Theorem 5.1 is easily deduced from Theorem 5.6. Similarly, if $\sigma(T) \cap \Gamma$ is finite and $R \sigma(T) \cap \Gamma=\varnothing$, Theorem 5.1 can be deduced from [1, Theorem 5].

We conclude this section by mentioning the relation of the discrete results with our Stability Theorem 2.4. It may be deduced from the discrete Theorem 5.1 
provided that the spectral mapping theorem holds in the form: $\sigma(T(t)) \cap \Gamma=$ $\exp (t \sigma(A)) \cap \Gamma$ for some $t>0$. However, the latter may fail even in situations where Theorem 2.4 is applicable as the following example shows.

EXAMPLE 5.5. Let $\Omega=\{z \in \mathbf{C :} 0<|z| \leq 1\}$ and $X=C_{0}(\Omega)$. Define $\tau$ by

$$
(T(t) f)\left(r e^{i \theta}\right)=e^{-r t} \cdot f\left(r e^{i(\theta+r / t)}\right) \quad(t \geq 0, r>0, \theta \in \mathbf{R}) .
$$

Then by [2, Theorem 4.4],

$$
\sigma(A)=\left[\{0\} \cup \bigcup_{n \in \mathbf{Z}}\{(-r+i n / r): n \in \mathbf{Z}, 0<r \leq 1\}\right],
$$

so $\exp (t \sigma(A)) \cap \Gamma=\{1\}$, but

$$
\sigma(T(t)) \cap \Gamma=\overline{\exp (t \sigma(A))} \cap \Gamma=\Gamma \text { for all } t>0 .
$$

It is easy to see that $\tau$ is stable (either directly or applying Theorem 2.4).

\section{REFERENCES}

1. G. R. Allan, A. G. O'Farrell and T. J. Ransford, A Tauberian theorem arising in operator theory, Bull. London Math. Soc. 19 (1987), 537-545.

2. W. Arendt and G. Greiner, The spectral mapping theorem for one-parameter groups of positive operators on $C_{0}(X)$, Semigroup Forum 30 (1984), 297-330.

3. N. Dunford and J. T. Schwartz, Linear operators, Part I, Wiley, New York, 1958.

4. A. E. Ingham, On Wiener'a method in Tauberian theorems, Proc. London Math. Soc. (2) 38 (1935), 458-480.

5. Y. Katznelson and L. Tzafriri, On power bounded operators, J. Functional Anal. 68 (1986), $313-328$.

6. J. Korevaar, On Newman's quick way to the prime number theorem, Math. Intelligencer 4 (1982), 108-115.

7. U. Krengel, Ergodic theorems, De Gruyter, Berlin, 1985.

8. R. Nagel (ed.), One-parameter semigroups of positive operators, Lecture Notes in Math., vol. 1184, Springer-Verlag, Berlin and New York, 1986.

9. D. J. Newman, Simple analytic proof of the prime number theorem, Amer. Math. Monthly $\mathbf{8 7}$ (1980), 693-696.

10. B. Sz.-Nagy and C. Foias, Harmonic analysis of operators on Hilbert space, North-Holland, Amsterdam, 1970.

11. D. V. Widder, An introduction to transform theory, Academic Press, New York, 1971.

12. M. Wolff, A remark on the spectral bound of the generator of a semigroup of positive operators with applications to stability theory, Functional Analysis and Approximation (Proc. Conf., Oberwolfach, 1980) (P. L. Butzer, B. Sz.-Nagy, E. Görlich, eds.), Birkhäuser, Basel, 1981, pp. 39-50.

13. D. Zagier, Short proof of the prime number theorem, unpublished manuscript.

\section{Mathematisches Institut DeR UniVersität, D-7400 Tübingen, West Germany}

ST. JohN's COllege, OXFord OX1 3JP, ENGLAND (Current address of C. J. K. Batty)

Current address (W. Arendt): Equipe de Mathématiques de Besançon, Université de FrancheComté, 25030 Besançon Cedex, France 